$\xi=-1$

\title{
Evaluating South Korean Based Mobile Role Playing Games with Playability Heuristic Evaluation
}

\author{
Louis Laja Uggah ${ }^{1 *}$, Ahmad Azaini bin Abdul Manaf ${ }^{2}$, Azaze-Azizi Abdul Adis ${ }^{3}$ \\ ${ }^{1}$ Institute of Design and Innovation, Universiti Malaysia Sarawak \\ ${ }^{2}$ Faculty of Applied and Creative Arts, Universiti Malaysia Sarawak \\ ${ }^{3}$ Faculty of Business, Economics and Accountancy, Universiti Malaysia Sabah \\ *Corresponding author E-mail: Louislaja83@gmail.com
}

\begin{abstract}
This research compared two role-playing games developed by South Korean-based company which is Netmarble Games. These games were selected based on their number of downloads among iOs mobile users. The Heuristic Evaluation method was selected to study this games based on 3 categories which are Mobility, Usability and Gameplay. In conclusion, this study has presented evaluation on these 2 games based on playability heuristic evaluation and have identified key areas to be improved by game designers.
\end{abstract}

Keywords: Role Playing Games; Mobile Games; Heuristic Evaluation; Usability; Game Play.

\section{Introduction}

The South Korean mobile game industry has grown and developed tremendously for the past decade. Mobile games are categorized under creative content industry which recorded an export rate of more than US $\$ 5$ billion by South Korea in 2014 and became among the most successful contributors to the country economy [1]. Mobile games can be divided into different types of genres such as strategy, sports, role-playing, and puzzle games. Almost two-thirds of smartphone users out of the 50 million of South Korean population play games on their phones. More than $90 \%$ of Korean smartphone users run Android and spend more time on their phones playing games [2]. Recently, South Korean based mobile games are facing strong competition from other Asian countries such as China, especially in MMORPH and role playing games. According to [3], South Korean computer games industry is struggling to compete with fast growing Chinese competitors.

The aims of the present study are divided into three parts, which are:

a) To conduct Heuristic Evaluation Playability (HEP) on mobile role-playing games developed by South Korean based production company.

b) To identify weaknesses and strengths of these mobile role playing games based on the HEP findings and analysis

Affiliation: Institute of Design and Innovation, University Malaysia Sarawak

c) To provide recommendations in terms of usability on improvements of these games based on the HEP analysis.

HEP is a set of evaluations to apply to heuristics to determine the playability of games, based on an extensive review of the litera- ture. A heuristic evaluation involves evaluating a product and provides positive and negative opinions about the product [4]. According to a study conducted by [5], they analyzed multiple heuristics to determine how effective they are in providing valid and effective evaluations of video games. Heuristics are a measure of usability. They are most often used for software design. However, heuristics for games have a slightly different purpose from heuristics for software design. In software design, a program should be easy to use and difficult to master, with an enjoyable user interface. Games should also be easy to use and have an enjoyable user interface, but they should be difficult to master. Therefore, the same heuristics cannot be used for software design and for digital games. Instead, new heuristics must be designed to be able to determine the playability of games.

A study conducted by [6] on the popular social media-based game "Farmville," based on several usability heuristics. Six evaluators played the game "Farmville," beginning with making a new account as any new player would have to, and noted whether the game met various requirements on a three-point scale (Yes, No, Other). These requirements were taken from the heuristics of [79], as the researchers felt that none of these heuristics alone had all the necessary information, but that combined they formed a total picture of game usability. The evaluators indicated whether "Farmville" met the requirements of the heuristics, thus providing an orderly piece of quantitative data as an evaluation.

This piece of research is novel in this review because it does not attempt to create or edit a new heuristic, or to study whether an existing heuristic is sufficient. The researchers felt that existing heuristics were not complete, but did not back this up with research; instead, they simply combined several pertinent heuristics to create the survey they used as their tool. Instead of evaluating a heuristic, this study used a heuristic to evaluate a game. Thus it indicates the usefulness of the heuristic from a game developer's point of view, as a checklist for developers to use to determine the usability of the game. However, it does not indicate whether the 
set of questions the researchers used was itself complete, or if there might be more usability issues with the game that were not covered in the heuristic.

This study also points to the fact that a game can be wildly successful even if it fails many points on a usability heuristic, as Farmville did indeed fail may questions on the usability survey but still has a massive base of players.

A definition of game usability was presented by [9] as "the degree to which a player is able to learn, control, and understand a game" (p. 1453). They distinguish usability from artistic and technical factors such as engagement, storyline, or performance. They build on the work of previous researchers such as [7] for creating design heuristics, but they point out that previous research on heuristics has focused on fun and engagement rather than usability. Therefore, [8] presented a heuristic for usability separate from the questions of whether a given game is fun and engaging.

A simple method was used by [9] of developing a usability heuristic: they looked at reviews of several different video games from the website GameSpot. They categorized the complaints that users reported about usability in order to create a list of the most common problems with usability based on these complaints, and a positively-worded checklist for designers to use as a heuristic to iteratively ensure that their games are playable, even when the games are not yet at a playable level to begin beta testing. It costs time and resources to ensure that a game meets every point on a heuristic as pointed by [10], so it may be not cost-effective for designers to focus on meeting every point in a heuristic when some of those points may not actually matter to the players, or for that genre of game.

\section{Methodology}

Mobile games present a different set of user needs than games that are meant to be played on a desktop or laptop. Mobile games have to be usable on a small touch screen, which changes both the visual design and the controls. Mobile game designers have to reconcile the production of a robust game that may have many different options and menus to choose from, with the small space of a mobile phone screen where extensive lists and options on the screen become clunky and make the game hard to play. The heuristics was examined by [11] necessary to ensuring a successful mobile game. Since this study was conducted in 2006, smart phones as we now know them were not part of the study. Therefore, there would now perhaps be a different set of heuristics, or at least new heuristic elements to consider for mobile games. Nevertheless, there are still specific heuristics that were important for mobile game players then, which are still important for mobile game players today. For example, designing the game to be considerate of people around is an important concept. In addition, [11] expressed that it is important for the game's user interface to be significantly different from the phone's user interface, so that users do not become confused. Validation had been conducted on these heuristics and evaluated five mobile games and the results concluded that these heuristics are useful in identifying playability problems in mobile games such game usability, mobility and playability problems [11] Some other elements were common between mobile phones and large computers, such as players being able to see their progress through the game easily, and tasks not being boring or repetitive. This research will be based on the final HEP which was validated by [11] below.

Table 1: Game usability heuristics [11]

\begin{tabular}{ll}
\hline No & Game Usability Heuristic \\
GU1 & Audio-visual representation supports the game \\
GU2 & Screen layout is efficient and visually pleasing \\
GU3 & Device UI and game UI are used for their own purposes \\
GU4 & Indicators are visible \\
GU5 & The player understands the terminology \\
GU6 & Navigation is consistent, logical, and minimalist \\
GU7 & Control keys are consistent and follow standard conventions
\end{tabular}

GU8 Game controls are convenient and flexible

GU9 The game gives feedback on the player's actions

GU10 The player cannot make irreversible errors

GU11 The player does not have to memorize things unnecessarily GU12 The game contains help

Table 2: Mobility heuristics [11]

\begin{tabular}{ll}
\hline No & Mobility heuristic \\
\hline MO1 & The game and play sessions can be started quickly \\
MO2 & The game accommodates with the surroundings \\
MO3 & Interruptions are handled reasonably \\
\hline
\end{tabular}

Table 3: Gameplay heuristics [11]

No Gameplay heuristics

The game provides clear goals or supports player created GP1 goals GP2 the results

GP3 The players are rewarded and rewards are meaningful

GP4 The player is in control

GP5 Challenge, strategy, and pace are in balance

GP6 The first-time experience is encouraging

GP7 The game story supports the gameplay and is meaningful

GP8 There are no repetitive or boring tasks

GP9 The players can express themselves

GP10 The game supports different playing styles

GP11 The game does not stagnate

GP12 The game is consistent

GP13 The game uses orthogonal unit differentiation

GP14 The player does not lose any hard-won possessions

Five games from iOS Store were selected for this study. These games fall in the role-playing game category. These two games from the Top 50 Grossing Games from iOS Store were selected because of their underperformance in terms of gross sales order to identify playability weaknesses in the game. The two games selected are (Game 1) Marvel Future Fight and (Game 2) Star Wars Force Arena. South Korean based production companies developed both of these games.

According to [12], three to five evaluators were recommended for heuristic evaluations but recently [13] recommended larger sample size of around 20 samples for quantitative studies. For this study, we have selected 20 evaluators to participate in this data collection process. All of the evaluators have experience in game evaluation and surveys. First of all the evaluators were asked to the play the games through an I-pad and evaluate these games based on the 3 heuristics tables. If a particular heuristic is followed by the game, ' $\mathrm{Y}$ ' is assigned or else ' $\mathrm{X}$ ' is assigned. The evaluation process was done at the meeting of Magna Glow Sdn Bhd, Kuching Sarawak.

\section{Results}

The results of the heuristic evaluation based on 3 evaluators are show in the table below.

Table 4: Heuristic Evaluation Overall Results

\begin{tabular}{llll}
\hline Game & Usability & Mobility & Gameplay \\
\hline Marvel Future Fight & $65.4 \%$ & $90 \%$ & $62 \%$ \\
Star Wars Force Arena & $69 \%$ & $65 \%$ & $45.3 \%$ \\
\hline
\end{tabular}

Table 5: Game usability results

\begin{tabular}{|c|c|c|}
\hline & GAME 1 & GAME 2 \\
\hline GU1 & $55 \%$ & $90 \%$ \\
\hline GU2 & $65 \%$ & $70 \%$ \\
\hline GU3 & $80 \%$ & $90 \%$ \\
\hline GU4 & $90 \%$ & $90 \%$ \\
\hline GU5 & $80 \%$ & $30 \%$ \\
\hline GU6 & $70 \%$ & $35 \%$ \\
\hline GU7 & $75 \%$ & $65 \%$ \\
\hline GU8 & $65 \%$ & $30 \%$ \\
\hline GU9 & $50 \%$ & $35 \%$ \\
\hline GU10 & $75 \%$ & $90 \%$ \\
\hline GU11 & $60 \%$ & $90 \%$ \\
\hline GU12 & $20 \%$ & $95 \%$ \\
\hline
\end{tabular}

Table 6: Mobility results 


\begin{tabular}{|l|l|l|}
\hline & GAME 1 & GAME 2 \\
\hline MO1 & $90 \%$ & $30 \%$ \\
MO2 & $95 \%$ & $95 \%$ \\
MO3 & $85 \%$ & $70 \%$ \\
\hline
\end{tabular}

ducing better stories which are meaningful in the gameplay for the players. According to [10], this type of category is important to keep the players interested with the game while [7] stated that this category addressed the value of a compelling supporting story and a realistic environment.

Table 7: Gameplay results GAME 1

\begin{tabular}{l|l|l|}
\hline & GAME 1 & GAME 2 \\
\hline GP1 & $45 \%$ & $30 \%$ \\
GP2 & $30 \%$ & $30 \%$ \\
GP3 & $75 \%$ & $30 \%$ \\
GP4 & $90 \%$ & $65 \%$ \\
GP5 & $50 \%$ & $30 \%$ \\
GP6 & $80 \%$ & $30 \%$ \\
GP7 & $90 \%$ & $25 \%$ \\
GP8 & $40 \%$ & $30 \%$ \\
GP9 & $20 \%$ & $35 \%$ \\
GP10 & $50 \%$ & $30 \%$ \\
GP11 & $70 \%$ & $65 \%$ \\
GP12 & $65 \%$ & $85 \%$ \\
GP13 & $75 \%$ & $85 \%$ \\
GP14 & $85 \%$ & $65 \%$ \\
\hline
\end{tabular}

\section{Analysis}

Based on the evaluation done by 3 evaluators, each heuristics were calculated in percentage base on each category. Table 4 above will show the overall result of the evaluation of these 2 games. Any category which achieved a result of below than $50 \%$ is considered an issue.

One of the issues highlighted by the evaluators for Game 1 is the lack of "Help" options in the game. If the option is actually available, the evaluators could not find it while doing the evaluations. While the HELP option is not available in Game 2 as well, the tutorials allow the player to understand the in-game easily before playing the game, therefore the HELP option is not essential. Majority of the evaluators (95\%) agreed that the tutorial is considered as the HELP option for Game 2. Game 2 achieved low ratings (30\%) for GU5 whereby the evaluators claim that they do not understand the game terminology. Low ratings in GU8 for Game 2 conclude that the game controls are not user friendly while low ratings in GU9 is cause by poor feedback in the game by the players' actions.

For the Mobility category, the evaluators identified that Game 2 has an issue when starting the game. Sometimes the gameplay is stuck and players have to restart the application all over again. The evaluators do not have any issues with MFF in terms of mobility.

As for the Gameplay category, both Game 1 and Game 2 did not fare very well. Game 1 achieved $62 \%$ of the overall points while Game 2 only achieved $45.3 \%$. The main issue highlighted by the evaluators for Game 1 were the ability of the gamers to express themselves in the game (GP9). As for Game 2, the issues were the the game were repetitive and boring (GP8), game story does not support the game play (GP7) and first time experience was not encouraging (GP6), game does not support different playing style (GP10).

\section{Discussion}

Heuristic evaluation shows that several weaknesses of these 2 games that need to be rectified in order to be competitive in the market. For Usability category, Game 1 does not contain proper Help function for the gamers. While tutorial is available in the beginning of the game, this can be easily forgotten by gamers when they play game. Accessible Help functions are recommended for both games to improve gamer's usability. For Mobility category (MO1), Game 2 has issues when starting the game and this can be fixed if the game designers are able to fix the bug, which cause the game to freeze while starting or playing the game. Both evaluators have issues with the Gameplay of these games especially with category (GP7) and this can be improved by proIn conclusion, this study has presented evaluation on these 2 games based on playability heuristic evaluation and have identified key areas to be improved by game designers. These results provide game designers information and identify the categories in which the game needs improvements for example Game 1 needs to improve in terms of gameplay while Game 2 needs to improve in terms of mobility and gameplay.

\section{Acknowledgement}

This work was supported by Universiti Malaysia Sarawak via Special Funding for Research Institutes (SpFRI), (104/SpFRI/427/6/4).

\section{References}

[1] Yong Jin D (2012), Hallyu 20: The new Korean wave in the creative industry. International Institute Journal University of Michigan 2(1).

[2] Monica G (2015), Here's Why Mobile Game Devs Have to Consider South Korea. Retrieved 7 January, 2017, from http://www.oneskyapp.com/blog/mobile-gaming-south-korea/

[3] Jung-A S (2016), South Korea Gaming Groups Struggle to Fend Off China. Retrieved January 5, 2017, from https://www.ft.com/content/c1696490-69f7-11e6-a0b1d87a9fea034f

[4] Nielsen J, Molich R (1990), Heuristic evaluation of user interfaces. CHI '90 Proceedings of the SIGCHI Conference on Human Factors in Computing Systems, 249-256.

[5] Desurvire H, Caplan M, Toth J (2004), Using heuristics to evaluate the playability of games. CHI '04 Extended Abstracts on Human Factors in Computing Systems, 1509-1512.

[6] Almeida S, Mealha O, Veloso A (2010), Interaction behavior of hardcore and inexperienced players: "Call of Duty: Modern Warfare" Context. Proceedings do SBGames 2010, 106-115

[7] Desurvire H, Wiberg C (2009), Game usability heuristics (PLAY) for evaluating and designing better games: the next iteration. OCSC '09 Proceedings of the 3d International Conference on Online Communities and Social Computing: Held as Part of HCI International, 557-566.

[8] Federoff M (2002), Heuristic and Usability Guidelines For The Creation and Evaluation of Fun in Video Games. Retrieved January 12, 2017, from http://ocw.metu.edu.tr/file.php/85/ceit706_2/10/MelissaFederoff_H euiristics.pdf

[9] Pinelle D, Wong N, Stach T (2008), Heuristic evaluation for games: usability principles for video game design. $\mathrm{CHI}$ '08 Proceedings of the SIGCHI Conference on Human Factors in Computing Systems, 1453-1462. doi:doi.org/10.1145/1357054.1357282

[10] Rodio F, Bastien J (2013), Heuristics for Video Games Evaluation: How Players Rate Their Relevance for Different Game Genres According to Their Experience. Retrieved January 11, 2017, from https://hal.inria.fr/hal-00877287/document

[11] Korhonen H, Koivisto E (2006), Playability heuristics for mobile games. Mobile HCI '06 Proceedings of the 8th conference on $\mathrm{Hu}$ man-Computer Interaction with Mobile Devices and Services, 9-16.

[12] Nielsen J (1995), How to Conduct a Heuristic Evaluation. Retrieved January 28, 24, from https://www.nngroup.com/articles/how-to-conduct-a-heuristicevaluation/

[13] Nielsen J (2012), How Many Test Users in a Usability Study?. Retrieved 22 June, 2017, from https://www.nngroup.com/articles/how-many-test-users/ 